\title{
Análise elementar de cinco plantas medicinais comercializadas no mercado do Ver-o-Peso em Belém Pará, Brasil
}

\begin{abstract}
Elemental analysis of five medicinal plants sold in the market Ver-o-Peso in Belém-Pará,
\end{abstract} Brazil.

\author{
Y. L. N. Sousa ${ }^{1}$; M. D. B. Silva ${ }^{2}$; K. G. F. Dantas 3 ; V. W. B. Diniz ${ }^{2 *}$ \\ ${ }^{1}$ Discente do Departamento de Ciência Naturais, Universidade do Estado do Pará, 6650-540, Belém-PA, Brasil \\ ${ }^{2}$ Docente do Departamento de Ciência Naturais, Universidade do Estado do Pará, 6650-540, Belém-PA, Brasil \\ ${ }^{3}$ Docente da Faculdade de Química/Grupo de Espectrometria Analítica Aplicada, Universidade Federal da Pará, \\ 66075110 Belém-PA, Brasil
}

*victor_bechir@yahoo.com.br

(Recebido em 06 de abril de 2016; aceito em 28 de abril de 2016)

\begin{abstract}
A humanidade faz uso de plantas medicinais para prevenção e o tratamento de diversas doenças desde a antiguidade até os tempos atuais, aproximadamente, $80 \%$ da população ainda utiliza esse recurso medicinal como um dos primeiros tipos de tratamento não convencional para cuidar da saúde. A população da Amazônia tem como hábito a utilização de várias plantas medicinais, por isso, este estudo teve como objetivo determinar a concentração de $\mathrm{Ca}, \mathrm{Fe}, \mathrm{Mg}, \mathrm{Mn}$ e $\mathrm{Zn}$ em cinco plantas medicinais e em seus chás, comercializadas no Mercado do Ver-o-Peso, localizado na cidade de Belém-PA. As plantas estudadas foram: carqueja (Baccharis trimera), chapéu de couro (Echinodorus grandiflorus), confrei (Symphytum officinale L.), erva doce (Pimpinela anisum) e losna (Artemisia absinthium L.). As amostras foram digeridas em forno de micro-ondas com cavidade e, posteriormente, analisadas por espectrometria de absorção atômica com chama (FAAS). Os resultados mostraram que nos chás, as plantas que apresentaram os maiores teores dos elementos foram a losna para o Ca e $\mathrm{Zn}\left(3668,40\right.$ e $17,03 \mathrm{mg} \mathrm{kg}^{-1}$, respectivamente), o chapéu de couro para o Fe e $\mathrm{Mn}\left(282,38\right.$ e $156,64 \mathrm{mg} \mathrm{kg}^{-1}$, respectivamente) e a erva doce para o $\mathrm{Mg}$ com $2108,50 \mathrm{mg} \mathrm{kg}^{-1}$. A análise elementar em plantas medicinais serve como um dado complementar referente à sua avaliação nutricional, com isso, neste estudo foi possível constatar que as plantas analisadas não apresentam risco de toxicidade quando ingerido os seus chás.

Palavras chave: Chá, Elementos, Plantas medicinais.
\end{abstract}

Humanity makes use of medicinal plants for prevention and treatment of various diseases since antiquity until the present time, approximately, $80 \%$ of the population still uses this medical resource as one of the first types of unconventional treatment for health care. The Amazon population has a habit of using various medicinal plants, so this study aims to determine the concentration $\mathrm{Ca}, \mathrm{Fe}, \mathrm{Mg}, \mathrm{Mn}$ and $\mathrm{Zn}$ in five medicinal plants and their teas, sold in the market Ver-o-Peso, in the city of Belem-PA. The plants were studied: Carqueja (Baccharis trimera), chapéu de couro (Echinodorus grandiflorus), confrei (Symphytum officinale L.), erva doce (Pimpinela anisum) and losna (Artemisia absinthium L.). The samples were digested in a microwave oven and subsequently analyzed by flame atomic absorption spectrometry (FAAS). Results showed that in teas, plants that have the highest levels of elements were losna for $\mathrm{Ca}$ and $\mathrm{Zn}$ (3668.40 e17.03 mg kg-1 , respectively), chapéu de couro for Fe and $\mathrm{Mn}$ (282.38 and $156.64 \mathrm{mg} \mathrm{kg}^{-1}$, respectively) and erva doce for $\mathrm{Mg}$ with $2108.50 \mathrm{mg} \mathrm{kg}^{-1}$. Elemental analysis of medicinal plants serves as a complementary data regarding its nutritional assessment, therefore, in this study it was possible to the plants analyzed no risk of toxicity when ingested their teas.

Keywords: Teas, Elements, Medicinal plants. 


\section{INTRODUÇÃO}

A humanidade faz uso de plantas medicinais para prevenção e o tratamento de diversas doenças desde a antiguidade até os tempos atuais. Cerca de $80 \%$ da população ainda utiliza esse recurso medicinal, utilizando-a como um dos primeiros meios alternativos de tratamento não convencional para o cuidado da saúde dos indivíduos e de suas famílias [1, 2, 3].

A concentração de um elemento em uma planta pode variar de acordo com a espécie, seu estágio de crescimento, condições do solo e sazonalidade. Entretanto, existe uma classe de plantas denominadas acumuladoras que podem apresentar elevadas concentrações de um ou mais elementos potencialmente tóxicos quando cultivadas em ambientes contaminados [4].

Os elementos metálicos contribuem para o equilíbrio do organismo, no entanto, em excesso, podem causar alguns problemas, como náusea, vômito, hipertensão e diarreia. A suplementação nutricional de minerais vem sendo cada vez mais frequentes na prescrição nutricional como suplemento, através de formulações a base de sais de metais ou metais quelados $[5,6,7]$.

No corpo humano, os constituintes químicos de plantas medicinais interagem diretamente ou indiretamente com a química do organismo humano. Uma vez que os constituintes ativos são absorvidos na corrente sanguínea, eles circulam influenciando o sistema para derivar os benefícios exigidos.

Os elementos como manganês (Mn), zinco ( $\mathrm{Zn})$, ferro (Fe) e outros, são de fundamental importância para a sobrevivência de plantas e animais, e a ausência desses nutrientes podem comprometer o ciclo de vida desses seres [8].

O interesse na composição química, levantamento bioquímico e prospecção mineral de produtos à base de plantas vêm crescendo, principalmente devido ao contínuo desenvolvimento dos setores de medicina, nutrição e farmacêuticos [9]. Com isso, torna-se de fundamental importância conhecer o potencial de influência que certos elementos possuem sobre os efeitos farmacológicos das drogas naturais obtidas a partir destas plantas [10].

$\mathrm{Na}$ cidade de Belém-PA, no mercado do Ver-o-Peso, pode-se encontrar barracas onde são comercializadas as mais diversas plantas medicinais para o tratamento de várias doenças. Entre as plantas mais procuradas pela população que frequenta este mercado, pode-se citar carqueja, chapéu de couro, confrei, erva doce e losna. Todas estas espécies são indicadas para tratar problemas gástricos.

A carqueja (Baccharis trimera) possui propriedades digestivas, antiúlcera e antiácida, as quais foram validadas em estudos com cobaias, ao mostrar que os extratos da planta reduziram a secreção gástrica e tiveram efeito analgésico e anti-inflamatório. O seu chá é indicado para tratamento de problemas hepáticos, contra disfunção estomacal e intestinal [11, 12].

O chapéu-de-couro (Echinodorus grandiflorus) é uma planta medicinal de grande expressão popular e tem propriedades adstringentes. Popularmente o seu chá é indicado para moléstias da pele e do fígado, colesterol alto, afecções do estômago, rins e bexiga e outras [13].

O confrei (Symphytum officinale L.) atualmente tem sido referenciado por suas propriedades antiinflamatória, cicatrizante, antipsóricas, anti-irritante e hidratante. O chá de confrei é utilizado para tratamento de úlceras do trato gastrointestinal, congestão pulmonar e inflamação $[14,15]$.

A erva-doce (Pimpinela anisum) possui diversas propriedades farmacêuticas, como inseticida, antifúngica, estimulante digestivo e estrogênica. Utiliza-se o chá de erva doce principalmente no tratamento de problemas digestivos, cólicas e distúrbios ginecológicos [16].

A losna (Artemisia absinthium L.) é muito utilizada na medicina tradicional por apresentar propriedades carminativas, diurética, colagoga, emenenagoga, abortiva e anti-helmitica e, recentemente, foi descoberta propriedades inseticida e herbicida, é também muito indicada para má digestão. O seu chá é usado no tratamento de azia, cólica intestinal e estimulante da digestão [17].

Sabe-se que nas plantas o Ca faz parte da molécula de um ou mais compostos orgânicos como pectato de cálcio. Este elemento é parte integrante da parede celular, conferindo a esta rigidez e controlando o seu crescimento [18]. A principal função do Fe é a ativação de enzimas, atuando no processo de fotossíntese, respiração, assimilação de $\mathrm{N}$ e $\mathrm{S}$, síntese de lignina e suberina e no metabolismo de auxina [19]. Mg, que é componente da molécula de clorofila, é 
essencial para as reações fotoquímicas e metabólicas das plantas e participa da ativação enzimática [20,18].

Mn é essencial à síntese de clorofila e sua função principal está relacionada com a ativação de enzimas, atua nos processos de fotossíntese, respiração, absorção iônica, controle hormonal e resistência a doenças. Participa também no metabolismo do nitrogênio e nos compostos cíclicos, como precursor de aminoácidos, fenóis e ligninas [21]. Zn atua como fator enzimático, é essencial para a atividade, regulação e estabilização da estrutura protéica ou uma combinação destas [22].

$\mathrm{Ca}$ é o elemento constituinte de ossos e dentes, no organismo aproximadamente $100 \%$ do $\mathrm{Ca}$ está presente no esqueleto, apenas uma pequena quantidade está presente nos fluídos corpóreos e nas células, entretanto, essa pequena quantidade é responsável pelo funcionamento adequado de nervos e músculos, pela coagulação sanguínea e controle de permeabilidade das membranas celulares. A carência desse elemento no organismo pode provocar osteoporose, raquitismo [23, 24].

Fe é um elemento essencial para seres humanos e animais e é um componente essencial da hemoglobina, sua carência no organismo é associada à anemia, provoca infecção gastrointestinal, hemorragia nasal e pode provocar infarto do miocárdio $[25,26] . \mathrm{Mg}$ desempenha um papel importante nas funções do corpo, na concentração adequada mantem a função renal e protegem os rins de danos [27]. Mn em quantidades adequadas é fundamental para a síntese normal e secreção de insulina, ele também atua como um cofator em sistemas enzimáticos [28].

$\mathrm{Zn}$ é o segundo microelemento mais abundante no corpo, presente nos tecidos, órgãos e fluidos corporais, ele não é armazenado em nosso organismo, por isso torna-se fundamental a ingestão diária desse elemento, pois ele é essencial para o desenvolvimento humano. E sua deficiência pode provocar falta de apetite, retardo no crescimento, lesões cutâneas, e outros. Porém, em concentrações elevadas, a toxicidade de $\mathrm{Zn}$ provoca náusea, vômitos, cefaléia e deficiência de cobre $[29,7,30]$.

A partir deste contexto, o presente estudo teve como objetivo analisar os elementos $\mathrm{Ca}, \mathrm{Fe}$, $\mathrm{Mg}, \mathrm{Mn}$ e Zn por espectrometria de absorção atômica em chama (FAAS), em cinco plantas medicinais (carqueja, chapéu de couro, confrei, erva doce e losna) comercializadas no Mercado do Ver-o-Peso, localizado na cidade de Belém-PA.

\section{MATERIAL E MÉTODOS}

As amostras estudadas foram adquiridas no Mercado do Ver-o-Peso, em barracas de venda de plantas medicinais, pois se buscou analisar aquelas que os consumidores mais fazem uso para tratamento de doenças estomacais. Com isso, foram selecionadas cinco plantas medicinais para serem estudadas: carqueja, chapéu de couro, confrei, erva doce e losna.

Após a aquisição das amostras no Mercado do Ver-o-Peso, as mesmas foram lavadas com água destilada e, em seguida, foram levadas para estufa a $40{ }^{\circ} \mathrm{C}$ para o processo de secagem até adquirirem peso constante, processo que levou $48 \mathrm{~h}$. Após obter peso constante, as amostras foram moídas com auxílio de gral e pistilo e, posteriormente, armazenadas em frascos de polietileno previamente descontaminados.

Para a digestão das amostras, uma massa de, aproximadamente, $0,25 \mathrm{~g}$ da amostra moída foi pesada e, em seguida, transferida para um frasco de digestão em triplicata $(\mathrm{n}=3)$. As amostras foram digeridas com $6 \mathrm{~mL}$ de ácido nítrico $(65 \% \mathrm{v} / \mathrm{v})$ e $2 \mathrm{~mL}$ de peróxido de hidrogênio (30\% $\mathrm{m} / \mathrm{m}$ ). A digestão das amostras foi realizada em um forno de micro-ondas com cavidade modelo Start E (Milestone, Sorisole, Itália) [31]. O programa de aquecimento utilizado no forno de microondas com cavidade está apresentado na Tabela 1. 
Tabela 1: Programa de aquecimento do forno de micro-ondas

\begin{tabular}{llll}
\hline Tempo $(\mathbf{m i n})$ & Função & Temperatura & Potência \\
\hline $\mathbf{0}-\mathbf{1 0}$ & Rampa & $0-200{ }^{\circ} \mathrm{C}$ & $1000 \mathrm{~W}$ \\
\hline $\mathbf{1 0}-\mathbf{3 0}$ & Patamar & $200{ }^{\circ} \mathrm{C}$ & $1000 \mathrm{~W}$ \\
\hline $\mathbf{3 0}-\mathbf{6 0}$ & Ventilação & $200-34{ }^{\circ} \mathrm{C}$ & - \\
\hline
\end{tabular}

Após o resfriamento foi observado que as amostras estavam parcialmente digeridas, dessa forma as mesmas foram centrifugadas por $20 \mathrm{~min}$ a $300 \mathrm{rpm}$, após esse processo, foi retirado o sobrenadante e reservado. Ao precipitado foi adicionado $2 \mathrm{~mL}$ de ácido fluorídrico e encaminhado para a mesa agitadora por $3 \mathrm{~h}$ e, em seguida, foi adicionado $1 \mathrm{~g}$ de ácido bórico e com auxílio de um agitador tipo vortex se fez a solubilização do ácido. Após este procedimento, os tubos foram centrifugados novamente por mais $20 \mathrm{~min}$ a $300 \mathrm{rpm}$. Ao término de todo o processo, essa solução foi adicionada ao sobrenadante, retirado no início, e aferida a $25 \mathrm{~mL} c o m$ água ultrapura. Este procedimento foi realizado em triplicata para cada amostra [32].

Os chás foram preparadas a partir de $1 \mathrm{~g}$ de material seco e, posteriormente, transferidas para um béquer de $100 \mathrm{~mL}$ ao qual foram adicionados $50 \mathrm{~mL}$ de água fervente. Após atingir a temperatura ambiente, cada chá foi filtrado em papel de filtro quantitativo. O branco analítico foi preparado pelo mesmo procedimento, porém, sem adição da amostra [32, 33].

Para determinação de $\mathrm{Ca}, \mathrm{Fe}, \mathrm{Mg}, \mathrm{Mn}$ e $\mathrm{Zn}$ nos digeridos e chás foi utilizado um espectrômetro de absorção atômica com chama (Modelo iCE 3300, Thermo Scientific, Cambridge, Reino Unido) equipado com corretor de fundo de lâmpada de deutério. Como fontes de radiação foram utilizadas lâmpadas de catodo oco. A mistura ar/acetileno foi utilizada como gás oxidante e combustível, respectivamente. Os parâmetros instrumentais utilizados no FAAS estão apresentados na Tabela 2. O limite de detecção (LOD) e o limite de quantificação (LOQ) foram calculados usando-se 10 medidas do branco analítico, onde o LOD $=3 \mathrm{x} \mathrm{s} / \mathrm{m}$ e o $\mathrm{LOQ}=10 \mathrm{x} \mathrm{s} / \mathrm{m}$, sendo s o desvio padrão das medidas do branco analítico e $\mathrm{m}$ a inclinação da curva analítica usada na determinação dos elementos estudados. A exatidão das medidas por FAAS foi avaliada através de estudos de adição e recuperação dos analitos, onde foram adicionadas alíquotas conhecidas dos mesmos nos digeridos e nos chás.

Tabela 2: Parâmetros instrumentais para análise elementar por FAAS

\begin{tabular}{cccc}
\hline Elemento & $\begin{array}{c}\text { Corrente da } \\
\text { Lâmpada (mA) }\end{array}$ & $\begin{array}{c}\text { Resolução } \\
\text { Espectral (nm) }\end{array}$ & $\begin{array}{c}\text { Comprimento } \\
\text { de Onda (nm) }\end{array}$ \\
\hline $\mathbf{C a}$ & 5 & 0,5 & 422,7 \\
\hline $\mathbf{F e}$ & 6 & 0,2 & 248,3 \\
\hline $\mathbf{M g}$ & 4 & 0,5 & 285,2 \\
\hline $\mathbf{M n}$ & 5 & 0,2 & 279,5 \\
\hline $\mathbf{Z n}$ & 5 & 1,0 & 213,9 \\
\hline
\end{tabular}

\section{RESULTADOS E DISCUSSÃO}

Os percentuais de recuperação obtidos foram adequados mostrando valores de 97,68\% para o $\mathrm{Ca}, 90,86 \%$ para o $\mathrm{Fe}, 98,40 \%$ para o $\mathrm{Mg}, 89,90 \%$ para o $\mathrm{Mn}$ e $97,74 \%$ para o $\mathrm{Zn}$, mostrando uma boa exatidão da metodologia proposta [32,33].

Os teores dos elementos nas amostras digeridas, nos chás e a percentagem de extração dos elementos são apresentados na Tabela 3. 
Tabela 3: Teores médios $\left(\mathrm{mg} \mathrm{kg}^{-1}\right)$ e seus desvios-padrão dos elementos nas plantas estudadas $(n=3)$.

\begin{tabular}{|c|c|c|c|c|c|c|}
\hline & ostras & $\mathrm{Ca}$ & $\mathbf{F e}$ & Mg & Mn & $\mathbf{Z n}$ \\
\hline \multirow{3}{*}{$\begin{array}{l}\text { Erva } \\
\text { Doce }\end{array}$} & Digerido & $\begin{array}{r}6258,01 \\
\pm 55,76\end{array}$ & $<\mathrm{LOD}^{\mathrm{a}}$ & $\begin{array}{c}3140,21 \\
\pm 8,45 \\
\end{array}$ & $<\mathrm{LOD}$ & $\begin{array}{r}34,04 \\
\pm 0,42 \\
\end{array}$ \\
\hline & Chá & $\begin{array}{l}1632,85 \\
\pm 68,31 \\
\end{array}$ & $<\mathrm{LOD}$ & $\begin{array}{l}2108,50 \\
\pm 51,83 \\
\end{array}$ & $<\mathrm{LOD}$ & $\begin{array}{r}15,94 \\
\pm 0,70 \\
\end{array}$ \\
\hline & \% Extração & 26,09 & $\mathrm{NC}^{\mathrm{b}}$ & 67,14 & $\mathrm{NC}$ & 46,83 \\
\hline \multirow{3}{*}{ Confrei } & Digerido & $<\mathrm{LOD}$ & $\begin{array}{c}2287,3 \\
6 \\
\pm 5,81\end{array}$ & $\begin{array}{c}2713,41 \\
\pm 130,96\end{array}$ & $\begin{array}{l}86,20 \\
\pm 3,70\end{array}$ & $\begin{array}{c}54,78 \\
\pm 1,80 \\
\end{array}$ \\
\hline & Chá & $<\mathrm{LOD}$ & $<$ LOD & $\begin{array}{l}554,32 \\
\pm 8,46 \\
\end{array}$ & $<\mathrm{LOD}$ & $\begin{array}{r}13,98 \\
\pm 0,19\end{array}$ \\
\hline & \% Extração & $\mathrm{NC}$ & $\mathrm{NC}$ & 20,42 & $\mathrm{NC}$ & 25,52 \\
\hline \multirow{3}{*}{ Carqueja } & Digerido & $\begin{array}{r}6636,28 \\
\pm 168,73 \\
\end{array}$ & $<\mathrm{LOD}$ & $\begin{array}{l}1934,60 \\
\pm 76,56 \\
\end{array}$ & $\begin{array}{r}464,63 \\
\pm 16,38 \\
\end{array}$ & $\begin{array}{r}76,98 \\
\pm 3,06 \\
\end{array}$ \\
\hline & Chá & $\begin{array}{l}2528,62 \\
\pm 90,31 \\
\end{array}$ & $<\mathrm{LOD}$ & $\begin{array}{l}297,40 \\
\pm 2,85 \\
\end{array}$ & $\begin{array}{l}21,41 \\
\pm 0,40 \\
\end{array}$ & $\begin{array}{r}7,90 \\
\pm 0,16 \\
\end{array}$ \\
\hline & \% Extração & 38,10 & $\mathrm{NC}$ & 15,37 & 4,60 & 10,24 \\
\hline \multirow{3}{*}{$\begin{array}{l}\text { Chapéu } \\
\text { de Couro }\end{array}$} & Digerido & $\begin{array}{r}7007,34 \\
\pm 264,36 \\
\end{array}$ & $\begin{array}{c}3002,1 \\
2 \\
\pm 85,63\end{array}$ & $\begin{array}{c}3121,76 \\
\pm 3,52\end{array}$ & $\begin{array}{l}1129,23 \\
\pm 21,92 \\
\end{array}$ & $\begin{array}{c}20,02 \\
\pm 0,15\end{array}$ \\
\hline & Chá & $\begin{array}{l}2206,77 \\
\pm 107,54 \\
\end{array}$ & $\begin{array}{l}282,38 \\
\pm 8,61 \\
\end{array}$ & $\begin{array}{l}1140,73 \\
\pm 51,50 \\
\end{array}$ & $\begin{array}{l}156,64 \\
\pm 6,02 \\
\end{array}$ & $<\mathrm{LOD}$ \\
\hline & \% Extração & 31,49 & 9,04 & 36,54 & 13,87 & $\mathrm{NC}$ \\
\hline \multirow{5}{*}{ Losna } & Digerido & $\begin{array}{r}45731,50 \\
\pm 1639,05 \\
\end{array}$ & $\begin{array}{l}388,58 \\
\pm 7,85 \\
\end{array}$ & $\begin{array}{r}7115,41 \\
\pm 322,41 \\
\end{array}$ & $\begin{array}{r}76,90 \\
\pm 0,82 \\
\end{array}$ & $\begin{array}{r}52,71 \\
\pm 2,23 \\
\end{array}$ \\
\hline & Chá & $\begin{array}{c}3668,40 \\
\pm 71,21 \\
\end{array}$ & $\begin{array}{c}20,75 \\
\pm 0,94 \\
\end{array}$ & $\begin{array}{r}1259,38 \\
\pm 32,34 \\
\end{array}$ & $<$ LOD & $\begin{array}{r}17,03 \\
\pm 0,54 \\
\end{array}$ \\
\hline & \% Extração & 8,02 & 5,31 & 17,69 & $\mathrm{NC}$ & 32,31 \\
\hline & $\begin{array}{c}\mathrm{LOD} \\
\left(\mathrm{mg} \mathrm{kg}^{-1}\right)\end{array}$ & 0,05 & 0,02 & 0,06 & 0,02 & 0,01 \\
\hline & $\begin{array}{c}\mathrm{LOQ} \\
\left(\mathrm{mg} \mathrm{kg}^{-1}\right)\end{array}$ & 0,19 & 0,07 & 0,22 & 0,04 & 0,03 \\
\hline
\end{tabular}

a < LOD: Abaixo do limite de detecção; ${ }^{\text {b }}$ NC: Não calculado.

Os valores para o Ca nas amostras digeridas variaram entre 6258,01 a $45731,50 \mathrm{mg} \mathrm{kg}^{-1}$. Estes valores encontrados estão dentro do intervalo obtido em amostras digeridas de plantas medicinais [32, 34, 35].

Para as análises de $\mathrm{Fe}$, os valores variaram entre 388,58 a $3002,12 \mathrm{mg} \mathrm{kg}^{-1}$. Pode ser observado que as amostras de erva doce e carqueja apresentaram valores abaixo do LOD, e as demais amostras apresentaram valores de acordo com os valores encontrados por outros autores $[36,37,38]$.

Os teores de $\mathrm{Mg}$ variaram entre 1934,60 a 7115,40 $\mathrm{mg} \mathrm{kg}^{-1}$, evidenciando-o que este elemento foi o que melhor se destacou nas plantas analisadas, dentre esses valores apenas a amostra de losna está fora do intervalo encontrado por outros autores, pois apresentou valores superiores $[39,40]$.

Mn apresentou teores com variação entre 76,89 a 1129,23 mg kg-1. Apenas a amostra de erva doce apresentou valores abaixo do LOD. As demais plantas apresentaram valores de acordo com os teores encontrados em outras plantas medicinais [41, 42, 43].

Os teores de $\mathrm{Zn}$ apresentaram valores que variaram entre 20,02 a $76,97 \mathrm{mg} \mathrm{kg}^{-1}$, que são concordantes com os teores de $\mathrm{Zn}$ em amostras de plantas medicinais [30, 44, 45]. 
Nos chás, os valores para o Ca variaram entre 1632,85 a 3668,40 mg kg-1. Estes valores estão dentro dos teores encontrados na literatura para chás de ervas medicinais [27, 46].

Para a determinação do Fe se obteve o intervalo de 20,74 a $282,38 \mathrm{mg} \mathrm{kg}^{-1}$, sendo que esses valores foram detectados apenas em chapéu de couro e losna. As amostras de carqueja, confrei e erva doce apresentaram teores abaixo do LOD para este elemento. Entretanto, os valores encontrados estão dentro da variação encontrada nos chás de plantas medicinais analisadas em outros estudos [47, 48, 49].

$\mathrm{Mg}$ foi o elemento presente em todos os chás das plantas analisadas, com os valores variando de 297,40 a $2108,50 \mathrm{mg} \mathrm{kg}^{-1}$. Porém, esses valores se encontram abaixo dos teores encontrados em outros trabalhos [50,51].

Os valores encontrados para o Mn variaram entre 21,40 a $156,63 \mathrm{mg} \mathrm{kg}^{-1}$, referente as amostras de carqueja e chapéu de couro, respectivamente. As amostras de erva doce, confrei e losna apresentaram teores abaixo do LOD. Os valores das amostras analisadas estão de acordo com a literatura $[52,53,54]$.

Para o $\mathrm{Zn}$, os valores variaram entre 7,89 a $17,03 \mathrm{mg} \mathrm{kg}^{-1}$, sendo concordantes com os valores encontrados por outros autores $[55,56]$.

As porcentagens de extrações foram calculadas a partir dos valores dos elementos nos chás, comparados com os teores dos mesmos nas amostras digeridas. As plantas que se mostraram melhor na ordem de extração foram a carqueja com $38,10 \%$ para Ca, chapéu de couro para Fe e Mn com teores na ordem de 9,04 e 13,87 \%, e erva doce para Mg e Zn com 67,14 e 46,83\%, respectivamente.

Deste modo, é possível observar que entre os elementos estudados, o $\mathrm{Mg}$ foi o elemento que apresentou os maiores teores nas plantas analisadas neste estudo, onde a sua extração variou entre 15,37 a 67,14\%, seguido de Zn (10,24 a 46,83\%), Ca (8,02 a 38,10\%), Mn (4,60 a $13,87 \%)$ e por último o Fe $(5,31$ a $9,04 \%)$.

Levando em consideração a quantidade diária de consumo de elemento recomendada para um adulto, foi realizada uma estimativa a partir dos dados da Tabela 3, onde se adotou um padrão de preparo dos chás, usando $1 \mathrm{~g}$ da planta seca para uma xícara de $200 \mathrm{~mL}$ [57]. Sendo assim, para o Ca que possui Índice Diário Recomendado (IDR) igual a $800 \mathrm{mg}$ conforme a Recommended Dietary Allowances (RDA) [58], uma xícara de chá das plantas analisadas, correspondem de 1,60 a 3,60 mg da quantidade total estimada para um adulto ingerir ao longo do dia. Para o Fe (IDR de $14 \mathrm{mg}$ ), os valores variaram de 0,02 a 0,28 $\mathrm{mg}$ do recomendado, referente aos chás de losna e chapéu de couro, respectivamente. $\mathrm{Mg}$ foi o elemento que se mostrou presente em todas as formas de análises das plantas estudadas, com o IDR igual a 300 mg, os chás variaram de 0,27 a 2,10 mg desse valor. Para o Mn, os chás variaram de 0,02 a 0,15 $\mathrm{mg}$ dos $5 \mathrm{mg}$ recomendado pelo IDR. Para o $\mathrm{Zn}$, a variação ficou entre 0,006 a $0,01 \mathrm{mg}$ do IDR cujo valor estipulado é $15 \mathrm{mg}$.

A partir dos resultados obtidos, se observa que as plantas que mais se destacaram na presença de todos os elementos estudados foi chapéu de couro e losna e as que apresentaram os maiores teores dos elementos estudados nos chás foram losna, chapéu de couro e erva doce, respectivamente.

Entre as plantas medicinais analisadas, foi observado que não há risco de toxicidade na ingestão na forma de chá, visto que, a quantidade de elemento encontrada e comparada com o IDR apresentou valores muito baixos para uma possível contaminação. 


\section{CONCLUSÃO}

A partir da análise elementar das cinco plantas medicinais estudadas, foi possível constatar que não há risco de contaminação por $\mathrm{Ca}, \mathrm{Fe}, \mathrm{Mg}, \mathrm{Mn}$ e $\mathrm{Zn}$ ingerindo apenas os chás dessas plantas medicinais. Entretanto, como há poucos estudos que avaliem os elementos nessas plantas, a realização de estudos aprofundados nessa área se torna eficaz para conhecer os benefícios e malefícios que essas plantas têm a proporcionar para seus consumidores.

A análise de elementos traço por espectrometria de absorção atômica com chama em plantas medicinais serve também como um dado complementar referente à avaliação nutricional das mesmas. Dessa forma, é necessário para a comunidade acadêmica que outros estudos com plantas medicinais possam ser explorados e repassados aos consumidores.

Os consumidores precisam ser informados de maneira clara e objetiva sobre a quantidade de elementos que cada planta possui, permitindo que sua ingestão diária esteja de acordo com a legislação, diminuindo assim, o consumo exagerado e um possível risco de futuras complicações provocadas pelo excesso ou carência desses elementos no organismo.

\section{AGRADECIMENTOS}

Ao PIBIC e ao GEAAp (Grupo de Espectrometria Analítica Aplicada) da UFPA (Universidade Federal do Pará) pela colaboração na realização das análises.

\section{REFERÊNCIAS BIBLIOGRÁFICAS}

1. Firmo WCA, Menezes VJM, Passos CEC, Dias CN, Alves LPL, Dias ICL, Neto MS, Olea RSG. Contexto histórico, uso popular e concepção científica sobre plantas medicinais. Cad. Pesq., São Luís. 2011; 18.

2. Oliveira ER, Menini Neto L. Levantamento etnobotânico de plantas medicinais utilizadas pelos moradores do povoado de Manejo, Lima Duarte - MG. Revista Brasileira de Plantas Medicinais. 2012; 14 (2): 311-320.

3. Yamashita CI. Estudo sobre os constituintes inorgânicos presentes em diferentes espécies da planta medicinal do gênero Caseira coletadas em regiões distintas da mata atlântica, SP [dissertação]. Instituto de Pesquisas Energéticas e Nucleares. Universidade do Estado de São Paulo; 2006. 130 p.

4. Rêgo JF. Amostragem de sólidos em espectrometria de absorção atômica de fonte contínua com alta resolução: análise de plantas medicinais. Instituto de Química de Araraquara. Universidade Estadual Paulista, 2013.

5. Brunken GS, Guimarães LV, Fisberg M. Anemia em crianças menores de 3 anos que frequentam creches públicas em período integral. Jornal de Pediatria. 2002; 78: 50-56.

6. Olga S. Fundamentos de toxicologia. 2. ed. São Paulo: Atheneu, 2003.

7. Andrade ÉCB, Alves SP, Takase I. Avaliação do uso de ervas medicinais como suplemento nutricional de ferro, cobre e zinco. Ciência Tecnologia de Alimentos, Campinas. 2005; 25: 591-596.

8. Lima VF, Merçon F. Metais pesados no ensino da química. In: Revista Química Nova na Escola. 2011; 33(4): 199 - 205

9. Karak T, Bhagat RM. Trace elements in tea leaves, made tea and tea infusion: A review. Food Research International. 2010; 43: 2234-2252.

10. Diaconu D, Diaconu R, Navrotescu T. Estimation of heavy metals in medicinal plants and their infusions, Universitatii "Ovidius" Constanta - Seria Chimie. 2012; 23 (1): 115-120.

11. Gamberini MT. Inhibition of gastric secretion by a water extract from Baccharis triptera Mart. Memórias do Instituto Oswaldo Cruz. 1991; 86(2): 137-9.

12. Gené RM, Marin E, Adzet T. Anti-inflamatory effect of aqueous extracts of three species of the genus Baccharis. Planta Medica. 1992; 58(6): 565-566.

13. Almeida ER. Plantas medicinais brasileiras: conhecimentos populares e científicos. São Paulo: Hemus, 1993. 341 p.

14. Pileggi M, Raimam PM, Micheli A, Beatriz S, Bobato V, Ação antimicrobiana e interação endofilica em SYMPHITUM OFFICINALE L. Biological and Health Scinces. 2002; 8 (1): 47-55.

15. Rode D. Comfrey toxicity revisited. Trends Pharmacol Sci. 2002; 23(11): 497-499. 
16. Kwon YS, Choi WG, Kim WJ, Kyung cKim W, Kim MJ, Kang WH, \& Kim C M.. Antimicrobial constituents of Foeniculum vulgare. Archibes of Pharmacal Research. 2002; 25(2): 154-157.

17. Gonzalez-Coloma A, Bailen M, Diaz CE, Fraga BM, Martínez-Díaz R, Zuñiga GE, \& Burillo J. Major componentes of Spanish cultivated Artemisia absinthium populations: Antifeedant, antiparasitic, and antioxidant effects. Industrial Crops \& Products. 2012; 37 (1): 401-407.

18. Faquin V. Nutrição mineral de plantas. Curso de pós-graduação "lato sensu" (especialização) à distância solos e meio ambiente. Universidade Federal de Lavras - UFLA, 2005.

19. Malavolta E, Leao HC, Oliveira SC, Lavres J, Moraes MF, Cabral CP, \& Malavolta M. Repartição de nutrientes nas flores, folhas e ramos da laranjeira cultivar natal.

Revista Brasileira de Fruticultura 2006; 28(3): 506-511.

20. Castro CRT, Garcia R, Carvalho MM, Freitas VP. Efeitos do Sombreamento na Composição Mineral de Gramíneas Forrageiras Tropicais. Revista Brasileira de Zootecnia. 2001; 30 (6): 1959-1968.

21. Melarato M, Panobianco M, Vitti GD, Vieira RD. Manganês e potencial fisiológico de sementes de soja. Ciência Rural, Santa Maria. 2002; 32 (6): 1069-1071.

22. Fernandes MS. Nutrição mineral de plantas, Viçosa: SBCS, 2006.

23. Herper HÁ, Rodwell VW, Mayes PA. Manual de química fisiológica. São Paulo: Atheneu, 1982.

24. Martin Jr DW, Mayes PA, Rodwell VW, Granner DK. Harpe's. Review oh biochemistry. 20. Ed. California: Lange Medical Publications, 1985.

25. Hunt Jr. Bioavailability of Fe, $\mathrm{Zn}$ and other trace minerals for vegetarian diets. American Journal of Clinical Nutrition. 1994; 78: 633-639.

26. Jiang R, Manson JE, Meigs JB. Body iron stores in relation to risk of type-2 diabetes in apparently healthy women. Journal of the American Medical Association. 2004; 291: 711-717.

27. Kolasani A, Xu H, Millikan M. Evaluation of mineral content of Chinese medicinal herbs used to improvekidney function with chemometrics. Food Chemistry. 2011; 1: 106-110.

28. Korc M. Manganese action on pancreatic protein synthesis in normal and diabetic rats. American Journal of Physiology. 1983; 245: 628-634.

29. Zalewski PD, Truong-Tran AiQ, Grosser D, Jayaram L, Murgia C \& Ruffin RE. “ Zinc metabolism in airway epithelium and airway inflammation: basic mechanisms and clinical targets. A review". Pharmacology \& Therapeutics. 2005; 105: 127.

30. Pereira RM. Determinação de constituintes inorgânicos em amostras de ervas e raízes medicinais por espectrometria de absorção atômica com chama. Instituto de Ciências Exatas. Universidade Federal de Minas Gerais, 2010.

31. Aires LM, Alves BSF, Fernandes KG. Determinação de Cobre, Ferro, Manganês e Zinco em frutas da região Amazônica. 35- Reunião Anual da Sociedade Brasileira de Química. 2012.

32. Mescouto CST, Dantas KGF. Avaliação dos constituintes químicos e fracionamento de elementostraço em solos de terra preta e terra mulata da região Amazônica. Tese de Doutorado - Universidade Federal do Pará, Instituto de Ciências Exatas e Naturais, Programa de Pós-Graduação em Química, Belém-PA, 2012.

33. Amarante CB, Silva JCF, Muller RCS, Muller AH. Avaliação da composição mineral do chá da folha senescente de montrichardia linifera (arruda) schott (araceae) por espectrometria de absorção atômica com chama (faas). Quim. Nova. 2011; 34 (3): 419-423.

34. Weber G, Konieczyński P, Anal. Bioanal. Chem. 2003; 375:10.

35. Rihawy MS, Bakraji EH, Aref S, Shaban R. Elemental investigation of Syrian medicinal plants using PIXE analysis. Nuclear Instruments and Methods in Physics Research B. 2010; 6: 34-9. doi:10.1016/j.nimb.2010.06.034.

36. Dghaim R. Khatib SAl, Rasool H, Khan MA. Determination of Heavy Metals Concentration in Traditional Herbs Commonly Consumed in the United Arab Emirates. Journal of Environmental and Public Health, 2015. http://dx.doi.org/10.1155/2015/973878

37. Tokalioglu S. Determination of trace elements in commonly consumed medicinal herbs by ICP-MS and multivariate analysis. Food Chemistry, 2012 http://dx.doi.org/10.1016/j.foodchem.2012.04.093

38. Kalny P, Fijałek ZB, Daszczuk A, Ostapczuk P. Determination of selected microelements in polish herbs and their infusions. Science of the Total Environment. 2007; 381: 99-104. doi:10.1016/j.scitotenv.2007.03.026

39. Cindrić IJ, Zeiner M, Glamuzina E. Gerhard Stingeder. Elemental characterisation of the medical herbs Salvia officinalis L. and Teucrium montanum L. grown in Croatia. Microchemical Journal. 2012. doi:10.1016/j.microc.2012.06.

40. Kumara A, Nairb AGC, Reddyb AVR, Garga AN. Analysis of essential elements in Pragya-peya-a herbal drink and its constituents by neutron activation. Journal of Pharmaceutical and Biomedical Analysis. 2005; 37: 631-638. 
41. Ansari TM, Ikran N, Najam-Ul-Haq M, Fayyaz I, Fayyas Q, Grafoor I, And Khalid N.. Essencial trace metal (zinc, mangneses, copper and iron) levels in plants of medicinal importance. Journal of Biological Science. 2004; 4 (2): 95-99.

42. Nookabkaew S, Rangkadilok N, And Satayavivad J. Determination of Trace Elements in Herbal Tea Products and Their Infusions Consumed in Thailand. J. Agric. Food Chem. 2006; 54: 6939-6944.

43. Konieczyński $\mathrm{P}$, Wesołowski M. Interrelationships among selected essential elements in medicinal plant raw materials and their water-extractable forms. Herba polonica. 2013; 59 (2): 11-15.

44. Basgel S, Erdemoglu SB. Determination of mineral and trace elements in some medicinal herbs and their infusions consumed in Turkey. Science of the Total Environment. 2006; 359: 82-89.

45. Marthe DB. Determinação de $\mathrm{Al}, \mathrm{Cu}, \mathrm{Fe}, \mathrm{Pb}$ e $\mathrm{Zn}$ em amostras de alcachofra e catuaba por espectrometria de absorção atômica. Universidade Federal de Minas Gerais, 2011.

46. Silva TV, Rêgo JF, De Moraes M, Cavalheiro AJ, Anchieta JGN, Raposo J, Luiz J. Multi-element determination of calcium, potassium and magnesium in medicinal plant by high-resolution continuum source atomic absorption spectrometry. Eclética Química. 2012; 37: 45-50.

47. Baranowska I, Srogi K, Włochowicz A, Szczepanik K. Determination of Heavy Metal Contents in Samples of Medicinal Herbs. Polish Journal of Environmental Studies. 2002; 11 (5): 467-471.

48. Fatima I, Waheed S, Zaidi JH. Elemental analysisof Anethum gravedlens, SismbriumIrio Linn and Veronia Anthelmintica seeds byinstrumentalneutronactivationanalysis. Applied Radiation and Isotopes, 2013 .doi.org/10.1016/j.apradiso.2012.09.022.

49. Franco MJ, Caetano ICS, Caetano J, Dragunski DC. Determinação de metais em plantas medicinais comercializadas na região de Umuarama-PR. Arq. Ciênc. Saúde UNIPAR, Umuarama. 2011; 15(2): 121-127.

50. Kumar A, Singh RP, Singh NP. Analysis of nutritional elements in Indian medicinal herbs used to cure general weakness. Natural Science. 2012; 4: 211-215. DOI: 10.4236/ns.2012.44032.

51. Aguilar GE, Cantarelli MA, Marchevsky EJ, Escudero NL, Jose M, Camina JM*. Multielemental Analysis and Classification of Amaranth SeedsAccording to Their Botanical Origin. Journal of Agricultural and Food Chemistry. 2011; 59: 9059-9064. DOI: 10.1021/jf202610t.

52. Gieldon AB, Rylko E. Estimation of metallic elements in herbs and Spices Available on the Polish Market. Pol.J. Environ. Stud. 2013; 22 (4): 1251-1256.

53. Lokhande R, Singare P, Andhale M. Study on Mineral content of Some Ayurvedic Indian Medicinal Plants by Instrumental Neutron Activation Analysis and AAS Techniques. Health Science Journal. 2010; 4 (3).

54. Ghani A, Ali Z, Islam T, Sanaulah S, Saeed S. Nutrient evaluation and elemental analysis of four selected medicinal plants of soon valley Khushab, Punjab, Pakistan. Park. J. Pharm. Sci. 2014; 27 (3): 597-600.

55. Subramanian R, Gayathri S, Rathnavel C, Raj V. Analysis of mineral and heavy metals in some medicinal plants collected from local market. Asian Pacific Journal of Tropical Biomedicine, 2012. Journal homepage:www.elsevier.com/locate/apjtb

56. Hameed, I, Hussain F. Proximate and elemental analysis of five selected medicinal plants of family Solanaceae. Pak. J. Pharm. Sci. 2015; 28 (4): 1203-1215.

57. ANVISA. Agência Nacional de Vigilância Sanitária. Formulário de Fitoterápicos da Farmacopéia Brasileira. Brasília: Anvisa. 2011; 1: 23-51.

58. BRASIL. Secretaria de Vigilância Sanitária. Recommended dietary allowances (RDA). Ingestão diária recomendada (IDR) de vitaminas, minerais e proteínas. Portaria $\mathrm{n}^{\mathbf{o}} 33$, de 13 de janeiro de 1998. Diário Oficial da República Federativa do Brasil, Brasília, Seção I-E, p. 5, jan. 1998. 